\title{
Functional complexity of hair follicle stem cell niche and therapeutic targeting of niche dysfunction for hair regeneration
}

\author{
Chih-Lung Chen ${ }^{1}$, Wen-Yen Huang ${ }^{1}$, Eddy Hsi Chun Wang ${ }^{2}$, Kang-Yu Tai ${ }^{3}$ and Sung-Jan Lin ${ }^{1,3,4,5,6^{*}}$ (D)
}

\begin{abstract}
Stem cell activity is subject to non-cell-autonomous regulation from the local microenvironment, or niche. In adaption to varying physiological conditions and the ever-changing external environment, the stem cell niche has evolved with multifunctionality that enables stem cells to detect these changes and to communicate with remote cells/tissues to tailor their activity for organismal needs. The cyclic growth of hair follicles is powered by hair follicle stem cells (HFSCs). Using HFSCs as a model, we categorize niche cells into 3 functional modules, including signaling, sensing and message-relaying. Signaling modules, such as dermal papilla cells, immune cells and adipocytes, regulate HFSC activity through short-range cell-cell contact or paracrine effects. Macrophages capacitate the HFSC niche to sense tissue injury and mechanical cues and adipocytes seem to modulate HFSC activity in response to systemic nutritional states. Sympathetic nerves implement the message-relaying function by transmitting external light signals through an ipRGC-SCN-sympathetic circuit to facilitate hair regeneration. Hair growth can be disrupted by niche pathology, e.g. dysfunction of dermal papilla cells in androgenetic alopecia and influx of auto-reacting $T$ cells in alopecia areata and lichen planopilaris. Understanding the functions and pathological changes of the HFSC niche can provide new insight for the treatment of hair loss.
\end{abstract}

Keywords: Hair follicle stem cell, Niche, Function, Alopecia, Alopecia areata, Lichen planopilaris, Androgenetic alopecia, Therapy

\section{Background}

Hair forms a barrier to protect skin from external insults as well as to keep the body from temperature loss. Human hair, especially human scalp hair, also has important ornamental functions that are essential for social communication and senses of well-being. Unwanted hair loss can pose psychosocial distress to affected individuals [1]. Hair regeneration depends on the activation of hair follicle stem cells (HFSCs) [2-4]. As the hair follicle (HF) is an integral part of skin [5], its growth and the activity of HFSCs are regulated by various nearby cells of the HFSC niche in the skin $[6,7]$. We categorize the component cells of the HFSC niche into 3 groups according to their functions, including signaling, sensing

\footnotetext{
* Correspondence: drsjlin@ntu.edu.tw

'Department of Biomedical Engineering, College of Medicine and College of Engineering, National Taiwan University, Taipei, Taiwan

${ }^{3}$ Genome and Systems Biology Degree Program, National Taiwan University and Academia Sinica, Taipei, Taiwan

Full list of author information is available at the end of the article
}

and message-relaying. We review how HFSC activity is regulated by different signaling cells and how sensing and message-relaying cells help HFs to initiate a regenerative attempt in face of local injury and external environmental changes. In diseased states, we discuss how the pathological changes of the niche lead to dysregulated hair growth. In addition, we discuss how the influx or emergence of non-preexisting cells within the HFSC niche affects hair growth and depletes HFSCs. We also highlight the therapeutic implications of niche pathology with an aim to prevent hair loss and to promote hair growth.

Hair follicle structure, hair cycle and HFSC

The HF is one of the few organs that undergo cyclic involution and regeneration throughout life $[5,6,8,9]$. Structurally, HF is an epithelial organ consisting of two main parts: an epithelial cylinder composed of keratinocytes and the mesenchymal cells of dermal papilla (DP) 


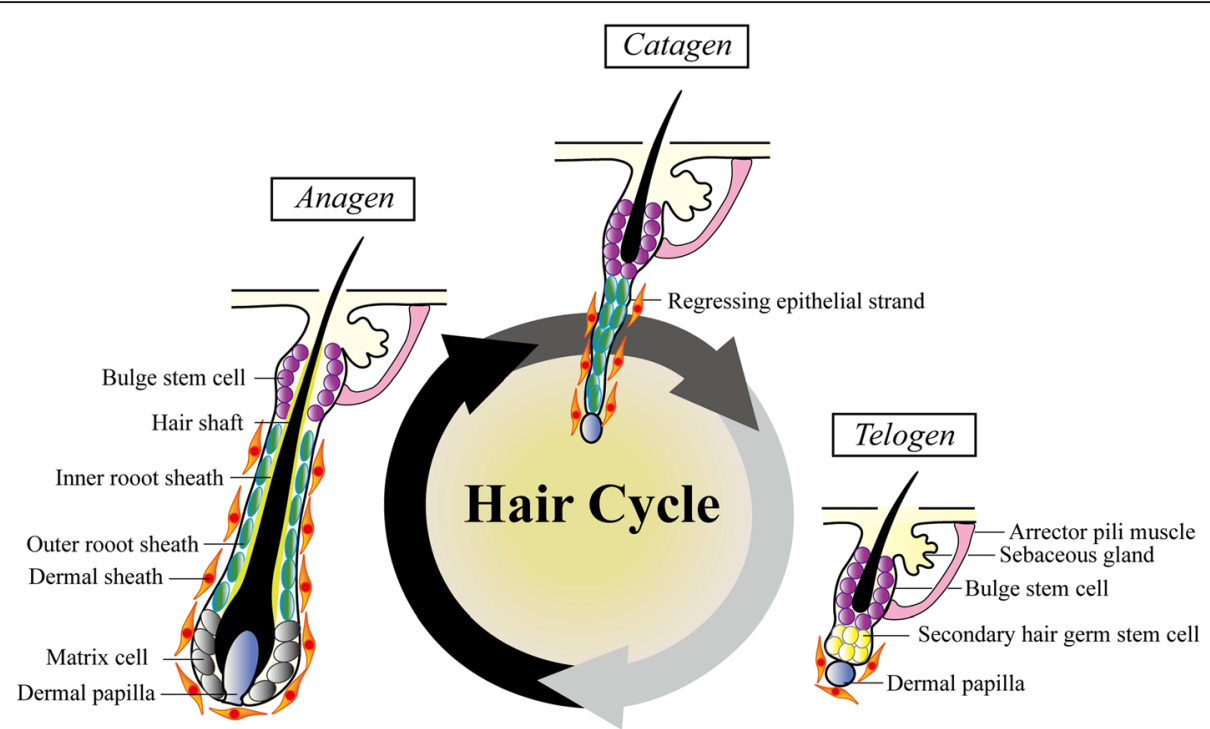

Fig. 1 Hair follicle structure, hair follicle stem cell and hair cycle. Quiescent HFSCS reside in the bulge region and primed HFSCS are located in the secondary hair germ. They are transiently activated in early anagen, giving rise to progeny that grow down to form the lower portion of HFs. HFs progress through catagen (regressing phase), telogen (resting phase) and anagen (growing phase) cyclically. Matrix cells in the hair bulb actively proliferate and differentiate to support the continued elongation of the hair shaft in anagen. In catagen, the hair bulb shrinks and the lower portion of the HF regresses through a progressively shortened epithelial strand into the telogen HF. In telogen, HFSCs in the secondary hair germ and bulge remain inactivated

and dermal sheath (Fig. 1) [5, 10]. During the hair cycle, HFs progress through anagen (growth), catagen (involution) and telogen (resting) phases and then re-enter anagen (Fig. 1) [5, 8-11]. Postnatal cycling and regeneration of HFs depend on sophisticated reciprocal epithelialmesenchymal interaction [6, 12-19].

Over the past 3 decades, progress has been made in understanding how the growth of HFs is regulated, particularly due to the discovery of HFSCs [2-4, 20-22]. HFSCs are first identified as slow-cycling label-retaining cells located in the bulge epithelium [2, 22]. In addition to this population of relatively quiescent stem cells, HFs harbor another population of primed stem cells with faster activation dynamics in the secondary hair germ of telogen HFs $[3,5,23]$. HF regeneration from telogen to anagen is fueled by the coordinated activation of these two cell populations: primed HFSCs in the secondary hair germ are first activated, followed by the activation of quiescent HFSCs in the bulge later [2-5].

\section{Signals and signaling cells within HFSC niche}

By definition, HF itself does not require the existence of surrounding niche cells to become a HF [24]. However, the integration of a variety of surrounding niche cells confer emergent functions on HFSCs, especially its ability to respond to changes of local, systemic and even external environments to begin a regenerative scheme or to remain quiescent. In diseased states, pathological infiltration of non-preexisting cells in the HFSC niche can lead to dysregulated hair growth. What constitutes the microenvironment that regulates HFSC activity and hair growth? Due to the continuous advance in hair research, more and more cell types (Fig. 2), including DP cells, adipose tissue, lymphatic vessels, nerves and immune cells, are identified to be contributing to the HFSC niche $[15,16,25-36]$, unveiling the complexity and sophistication in the interaction of HFSCs with its environment. Since activating and inhibitory signals can both be present in the HFSC niche, the probability of HFSC activation is the readout of the summation of both activating and inhibitory signals $[37,38]$. The two major counteracting signals are the bone morphogenetic protein $(\mathrm{BMP})$ and $\mathrm{Wnt} / \beta$-catenin signaling pathways $[28$, 37, 39]. High BMP signaling keeps HFSCs in an inactivated state, while $\mathrm{Wnt} / \beta$-catenin signaling promotes HFSC activation and maintains HF growth [17, 28, 3740]. Moreover, the TGF- $\beta 2$, Foxp1 and oncostatin $M$ signaling pathways have also been shown to regulate hair cycle $[16,29,37,41]$. Factors that are able to tilt the balance of $W n t / \beta$-catenin and BMP signaling can modulate HFSC activity, thereby suppressing or promoting anagen entry [6, 16, 29, 38, 42].

\section{Functional categorization- signaling, sensing and message-relaying modules in HFSC niche}

In addition to niche cells that provide either activating or inhibitory signals in the physiological state, niche cells of other functions also exist, enabling HFSCs to sense 


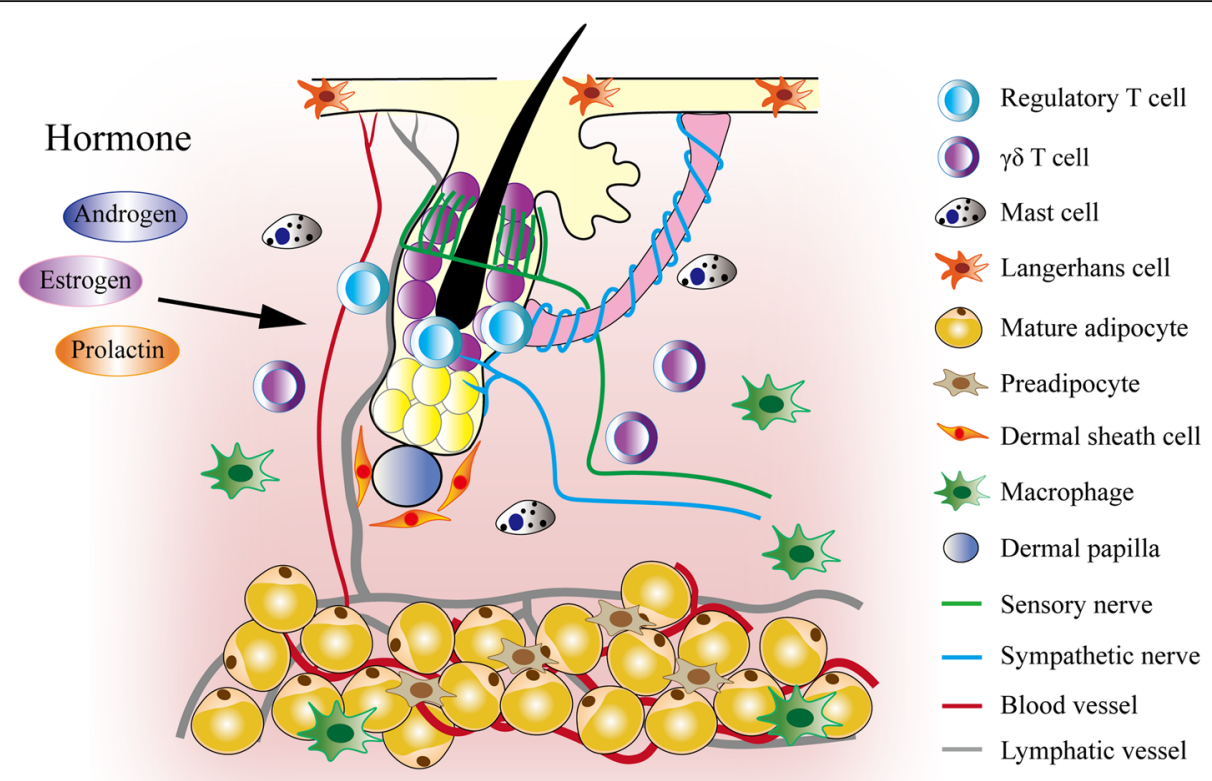

Fig. 2 Hair follicle stem cell niche. The HFSC niche is composed of various component cells, such as dermal papilla, preadipocytes, adipocytes, immune cells and nerves. Systemic hormones also regulate HFSCs directly or indirectly through the HFSC niche cells. Both activating and suppressive signals are present within the HFSC niche. The probability of HFSC activation depends on the summation of all the activating and inhibitory signals

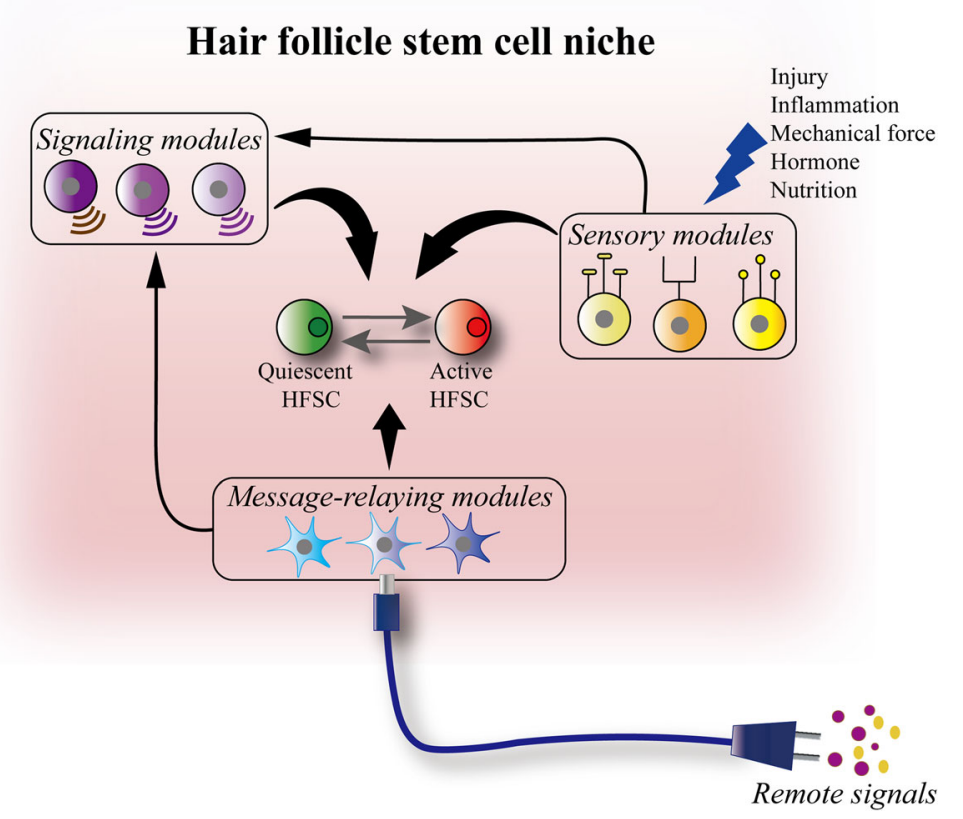

Fig. 3 Functional categorization of HFSC niche cells. According to the functions of niche cells, they are categorized into 3 groups: signaling modules, sensing modules, message-relaying modules. These functionally distinct modules are assembled into a multifunctional niche. Signaling modules regulate HFSC activity via cell-cell contact or paracrine secretion. Sensory modules detect environmental cues. Message-relaying modules transmit signals from remote cells/tissues to HFSCs. Sensory modules and message-relaying modules can directly signal to HFSCs or indirectly regulate HFSC activity through the signaling modules 
local, systemic and even external environmental changes to adjust their activity to meet local and organismal needs [28, 34, 35, 43-45]. Since additional cells of varied functions can be incorporated singly or in combination into the HFSC niche, we think that the niche cells can be modularized and these modules can be co-opted to construct the niche (Fig. 3). This is analogous to the design of a spaceship. The combination of different functional modules increases the functionality of the spaceship. Message-relaying modules allowed the communication between the Apollo 11 and NASA space center. The lunar module endowed Apollo 11 with an important function to land human on the moon. From this perspective, we divide HFSC niche cells into 3 functional modular categories: signaling, sensing and message-relaying (Fig. 3). Signaling cells directly provide activating or inhibitory signals for HFSCs through ligand secretion or cell-cell contact. Sensing cells detect the changes of local environmental cues and then directly or indirectly instruct HFSCs to remain quiescent or become activated. Message-relaying cells are capable of transmitting remote signals to the HFSC niche and then directly or indirectly modulate HFSC activity. Of note is that a single niche cell type can exhibit more than one function. We speculate that the co-option of various functional modules within a stem cell niche enables the animals to adapt their regenerative activity to the changing environment and to the altering physiological needs, thereby ameliorating the organismal fitness during evolution.

\section{Signals from dermal papilla for signaling and gain of testosterone-processing function in androgenetic alopecia}

DP cells are an essential signaling component within the HFSC niche (Fig. 2). Epithelial-mesenchymal interaction is indispensable not only for embryonic HF morphogenesis but also for postnatal hair cycling [12, 13, 36, 46] . In the embryonic stage, interaction with the specialized mesenchymal niche of HF dermal condensate/papilla stimulates and instructs the epithelium to sequentially form placode, germ, and peg [46]. For postnatal follicular epithelial-mesenchymal interaction, although DP cells provide signaling ligands, such as TGF- $\beta 2$ and FGF-7 [3, 16], to activate HFSCs for a new hair cycle, signals from epithelial cells are also required for proper anagen entry [17]. After HFs reach mature anagen, DP behaves as an instructive niche by regulating the proliferation and spatially ordered differentiation of transit-amplifying progenitors for proper hair shaft elongation and hair bulb structure maintenance [47]. Continued activation of Wnt/ $\beta$-catenin signaling in DP through epithelialmesenchymal interaction is indispensable for anagen progression [48]. During catagen, DP is also essential for the recession of the epithelial cylinder through controlled apoptosis/cell death [12]. In addition to physiological cycling, DP might also instruct the anagen repair process to avoid catagen entry when HFs are injured by chemo- and radiotherapy $[49,50]$.

Androgens are an important regulator for hair growth with paradoxical effects on HFs in different body regions. Androgens can stimulate the transformation of small vellus HFs into large terminal HFs after puberty, such as beard, pubic hair and axillary hair [51, 52]. On the contrary, in the scalp of genetically predisposed individuals of androgenetic alopecia or male pattern baldness, androgens inhibit hair growth, leading to progressive HF miniaturization [53]. Hyperandrogenism in females can lead to hirsutism with excessive male pattern hair growth [54]. These paradoxical effects of androgens on human hair growth have long been a puzzle $[55,56]$. Androgens act through the intracellular androgen receptor. In HFs, androgen receptors are mainly expressed by DP $[57,58]$. In contrast, keratinocytes do not express androgen receptors or show androgen receptor-dependent signaling activation, suggesting that keratinocytes may not be the primary responding cells in HFs $[59,60]$.

Alopecia due to HF aging is characterized by progressive HF atrophy with hair shaft miniaturization, prolonged telogen, and even loss of the entire HFs, resulting in diminished hair amount $[61,62]$. In male, androgenetic alopecia is the most common disease of premature HF aging. With genetic predisposition in affected individuals, DP cells in the balding area exhibit higher activity of type II 5alpha-reductase, an enzyme that are normally highly expressed in the prostate [58]. This enzyme converts testosterone into dihydrotestosterone through $5 \alpha$-reduction of testosterone [57]. Dihydrotestosterone is a more potent androgen with a higher affinity than testosterone [63]. Local sustained dihydrotestosterone stimulation to DP compromises its functions, leading to deteriorating hair growth, shortened anagen and prolonged telogen $[55,56]$. Therefore, the inappropriate gain of function, i.e. $5 \alpha-$ reducing ability to process testosterone, of these niche signaling cells in a patterned distribution is the primary cause of androgenetic alopecia.

DP cells from the balding scalp of androgenetic alopecia patients exhibit signs of senescent characters, such as loss of replicative potential, changes in cell size and shape, decrease or loss of is characteristic markers/molecular signature $[64,65]$. Although the mechanisms are not fully clarified yet, dihydrotestosterone seems to induce premature senescence in DP due to persistent androgen receptor activation. The balding DP cells not only lose the ability to promote HFSC proliferation but also produce inhibitory factors that suppress HFSCs and disrupt keratinocyte proliferation [66-69]. For example, 
Wnt signaling is critical for anagen entry and anagen progression $[17,40]$. Dkk1, a negative regulator of Wnt signaling, is overexpressed by balding DP cells [68]. Increased secretion of TGF- $\beta 1$ from DP in catagen promotes anagen-to-catagen transition [12, 70]. TGF- $\beta 1$ production is upregulated in balding DP and can compromise keratinocyte proliferation [69]. Additionally, balding DP cells also produce higher inflammatory cytokines, such as IL-6 [66, 67, 71]. IL-6 not only inhibits anagen entry but also disrupts normal anagen progression $[66,67,71]$. As a key mesenchymal signaling component in HFSC niche, targeted restoration of the normal signaling functions of DP cells can be an important strategy for the treatment of alopecia.

Currently, the most effective treatment for androgenetic alopecia is to suppress local dihydrotestosterone production by inhibiting 5 - $\alpha$-reductases. Finasteride and dutasteride are $5 \alpha$-reductase inhibitors with different specificity and potency [72, 73]. Finasteride mainly inhibits the type-II $5 \alpha$-reductase, the main $5 \alpha$-reductase subtype in HFs, while dutasteride suppresses both type-I and type-II $5 \alpha$-reductases. Long-term treatment with finasteride or dutasteride promotes hair growth in patients with androgenetic alopecia [74, 75]. Another FDA-approved medication for treating baldness is minoxidil [76, 77]. Minoxidil is a potassium channel opener originally designed for the treatment of hypertension [78]. Though the mechanisms are still unclear, it is speculated to promote hair growth through its effects on blood vessels or potassium channels [79].

\section{Mast cells, regulatory $\mathrm{T}$ cells, dendritic epidermal T cells}

The HF maintains its own distinctive immune system, and the interplay between HFs with immune cells ensures proper hair growth and protection against autoimmunity [30, 35, 80, 81]. The immune cells, including macrophages, mast cells, and $\mathrm{T}$ cells, modulate the activity of HFSCs (Fig. 2) [30-32, 35]. Mast cells are found in the perifollicular compartment of the HF [80]. While the role of mast cells in HFSC activation and differentiation is still unclear, histochemical and ultrastructural analysis in the murine skin showed a high level of degranulation during late telogen to early anagen transition and late anagen to early catagen transition [32, 82]. Several molecules secreted by mast cells could contribute to HF turnover, including histamine and serotonin which promote epidermal keratinocyte proliferation in situ [83]. Mast cell activity is also suspected to contribute to hair loss disorders, such as androgenic alopecia and cicatricial alopecia [84-86].

Regulatory $\mathrm{T}$ cells (Tregs) have been shown to reside in the HFSC epithelium and are in close contact with HFSCs [31]. Tregs can augment HFSC proliferation and differentiation following hair plucking injury through
Jagged1 (Jag1, 31]. The roles of Langerhans cells and dendritic epidermal $\mathrm{T}$ cells (DETC; $\gamma \delta \mathrm{T}$ cells), which are skin-resident antigen presenting cells and $\mathrm{T}$ cells respectively, in modulating HFSC activities are less defined [80]. Langerhans cells and DETCs are found in the outer root sheath of HFs [80]. The roles of DETCs on HFSCs have been reported in the context of wound healing. Activated DETCs not only stimulate epidermal stem cell proliferation to accelerate wound healing [87], but also favorably promote HFSC activation for hair regrowth [88].

\section{Macrophages for signaling and for injury- and force- sensing}

Physiologically, clusters of skin-resident macrophages can be found in the perifollicular compartment and have been implicated in the regulation of hair cycles $[30,89]$. The number of skin-resident $\mathrm{CD}_{11 \mathrm{~b}^{+} \mathrm{F} 4 /}$ $80^{+} \mathrm{Gr}^{-}$macrophages decreases due to apoptosis prior to the onset of anagen [30]. Upon their apoptosis, they release stimulatory factors, such as Wnt7b and Wnt10a, which promote HFSC activation and differentiation [30]. More recently, it was reported that a different subset of TREM2 ${ }^{+}$dermal macrophages (trichophages) have an inhibitory effect on hair growth [29]. This study stemmed from the discovery that inhibition of JAK-STAT signaling promoted hair growth via disrupting the maintenance of HFSC quiescence [90]. Mechanistically, oncostatin $M$ acts upstream of JAK-STAT5 signaling to maintain HFSC quiescence and oncostatin $\mathrm{M}$ is produced by TREM2 ${ }^{+}$ macrophages [29]. Depletion of this specific subset of macrophages leads to premature anagen entry [29].

Macrophages also exhibit other functions, including the sensing of skin injury and mechanical force. Wounding promotes premature anagen entry in skin $[44,45]$. When skin is wounded, macrophages are recruited and activated through the apoptosis signal-regulating kinase 1 (ASK1) [45]. Injury to HFs by hair plucking is also a potent stimulation to HFSCs. Injured by hair plucking, HFs recruit macrophages via the release of CCL2 [35]. TNF- $\alpha$ released by activated macrophages activates HFSCs by inducing AKT-dependent $\beta$-catenin accumulation [91]. Therefore, macrophages here capacitate the HFSC niche to sense the injuries to HFs or injuries to the surrounding skin to mount a regenerative attempt for skin protection. Additionally, macrophages also mediate the sensing of mechanical cues. Stretching skin can polarizes macrophages toward a M2 phenotype [92]. Pro-regenerative M2 macrophages stimulate hair regeneration via paracrine secretion of IGF and HGF. This demonstrates a mechanical force-macrophage axis in the regulation of tissue regeneration. Since there are multiple populations of macrophages within skin, each with 
distinct roles in the modulation of HFSC activation and differentiation, targeting macrophages can be a future direction for the management of hair loss.

\section{Influx of auto-reacting T-cells into HFSC niche disrupts hair growth in alopecia areata and lichen planopilaris}

Proper HF cycling is strongly dependent on the homeostasis in the maintenance of HFSCs as well as intact immune privilege [81]. Collapse of immune privilege as a result of environmental factors or genetic predisposition puts HFs in risk of immune/inflammatory attack $[81,93,94]$. An active immune response with the secretion of inflammatory cytokines such as interferon $-\gamma$ and TNF- $\alpha$ can certainly disrupt proper maintenance of HFSCs, leading to alopecia $[94,95]$. These cytokines are secreted in abundance by lymphocytes that are not usually present in the physiological state, including CD4 and CD8 T cells ( $\alpha \beta$ T cells) that surround or infiltrate the HF $[94,96]$.

One of the most common immunity-mediated alopecia is alopecia areata. Alopecia areata is an autoimmune form of hair loss that may be patchy on the scalp or progress into total body hair loss [97]. Alopecia areata is reversible, indicating that HFSCs are not lost during autoimmune/inflammatory attacks [97]. However, the exact etiopathogenesis of alopecia areata has not been completely elucidated. The development of alopecia areata is associated with the collapse of HF immune privilege which subsequently increases antigen presentation to surveying $\mathrm{T}$ cells that recognize HF epithelial and/or melanocyteassociated antigens as foreign, and mount autoimmune responses against HFs [93, 97-100]. There are ongoing investigations trying to identify the exact HF antigen and antigen-specific $\mathrm{T}$ cells involved in the onset of alopecia areata [99, 101]. The autoimmune attack does not kill HFSCs specifically, but, instead, the lower transient portion of anagen HFs [97]. Since HFSCs are preserved, removal of these pathogenic $\mathrm{T}$ cells from HFSC niche restores hair growth. Due to the wide variation of clinical presentation, such as numbers and extent of lesions, age of onset, duration of disease persistence, and unpredictable responses to treatment, there is still a lack of universal guidelines for the treatment of alopecia areata. Topical or intralesional steroids are favored in patients with limited diseases and topical minoxidil can be employed as an adjuvant therapy [102-104]. In patients with extensive hair loss, systemic steroids and other immunosuppressants, such as methotrexate, can be considered. Additionally, immunotherapy with repeated topical application of contact sensitizers, such as diphenylcyclopropenone (DPCP), has also been employed in patients with extensive hair loss [102, 105, 106].
Dysregulation of Tregs have also been suggested to be associated with the collapse of HF immune privilege in alopecia areata [31, 107, 108]. A defect or lack of Tregs could lead to unchecked autoimmune attack on HF cells [108]. Improvement of alopecia areata was also reported (hair regrowth and reduction of CD4 and CD8 T cells) by treating the patients with low-dose IL2 to promote recruitment of Tregs into the skin [109].

Recently, it was shown that a population of $\mathrm{CD}^{+} /$ $\mathrm{NKG}_{2} \mathrm{D}^{+} \mathrm{T}$ cells is necessary and sufficient for the development of alopecia areata $[107,110,111]$. The IFN- $\gamma$ response and several $\gamma$-chain $(\gamma c)$ cytokines are significantly upregulated in alopecia areata skin which can activate cytotoxic $\mathrm{CD}^{+} / \mathrm{NKG}^{2} \mathrm{D}^{+} \mathrm{T}$ cell infiltration. Using anti-INF- $\gamma$ antibody can efficiently block $\mathrm{CD}^{+} / \mathrm{NKG}^{+} \mathrm{D}^{+}$ $\mathrm{T}$ cell infiltration and prevent alopecia areata development in the mouse model [110]. Through this research, the authors identified a small molecule inhibitor that can effectively block JAK-STAT signaling important for $\mathrm{CD}^{+} / \mathrm{NKG} \mathrm{D}^{+} \mathrm{T}$ cell function and reverse alopecia areata in both the mouse model and human patients [110]. This research subsequently led to two successful clinical trials repurposing FDA-approved JAK inhibitors, ruxolitinib (JAK1/2 specific) and tofacitinib (pan-JAK), to treat moderate to severe alopecia areata [112, 113]. Because of this, more clinical trials have started with the aim to optimize treatment of alopecia areata with JAKspecific inhibitors and different routes of administration [114]. As described above, inhibiting JAK-STAT signaling may have a direct impact on hair cycle. While alopecia areata-affected mice were treated, it was observed that the mice grew fuller hair. When the treatment was applied to wild-type mice at telogen, the mice entered anagen faster and grew fuller and darker hair [90]. These observations implicate a dual role of JAK inhibitors in alopecia areata by inhibiting $\mathrm{CD}^{+} / \mathrm{NKG}^{2} \mathrm{D}^{+} \mathrm{T}$ cells and promoting HFSC proliferation or differentiation.

Hair loss in lichen planopilaris, also a chronic inflammatory disease of HFs, is irreversible with a final scarring change [94, 115]. Lichen planopilaris usually runs a slowly progressive course, presenting with single or multiple patches of perifollicular erythema, scaling, follicular hyperkeratosis and eventual loss of HFs [116]. In contrast to alopecia areata in which cytotoxic $\mathrm{T}$ cells target the hair bulb, lichen planopilaris is characterized by Th1-biased cytotoxic $\mathrm{T}$ cell infiltration around the bulge region where HFSCs reside. It is postulated that a selective collapse of immune privilege in the HFSC niche, possibly triggered by interferon- $\gamma$, contributes to the pathogenesis of lichen planopilaris [94]. Chronic niche inflammation might deplete HFSCs by directly inducing HFSC apoptosis or indirectly altering the niche environment to a state unfavorable for the maintenance of HFSCs [94, 117]. Depletion of HFSCs leads to loss of 
entire follicular structures. Therapeutically, there is also a lack of consensus for the treatment of this disease. Current treatment mainly relies on immunosuppressants, such as topical, intralesional or systemic steroids, hydroxychloroquine, cyclosporine and mycophenolate mofetil $[116,118]$. Prevention of the collapse of immune privilege of the HFSC niche can be a future direction for the treatment and prevention of this disease [94].

\section{Signals from adipose tissue and nutritional sensing}

Dermal white adipose tissue is a highly dynamic tissue in skin and the thickness oscillates during hair cycles [27, 119-121]. The dermal white adipose tissue becomes thickened from telogen to anagen and then decreases in thickness from anagen to catagen transition [120]. The increase of dermal white adipose tissue thickness during telogen to anagen transition is mainly contributed by proliferation and differentiation of preadipocytes and hypertrophy of maturate adipocytes [27, 122]. The maturation of preadipocytes with increased adipogenesis is dependent on epidermal Wnt/ $\beta$-catenin and sonic hedgehog $(\mathrm{SHH})$ signaling $[123,124]$. Epidermal Wnt/ $\beta$ catenin signaling is a signaling cascade initiator that is required for dermal adipocyte differentiation [123]. After anagen is initiated, the increased production of $\mathrm{SHH}$ by HF transit-amplifying cells promotes adipogenesis in preadipocytes via peroxisome proliferator-activated receptor $\gamma$ [124]. How the dermal white adipose tissue thickness is reduced during anagen to catagen transition is still unclear. Since no apoptosis of mature adipocytes is detected [27], it is possible that adipocytes might undergo dedifferentiation through a lipolytic or autophagic process [121]. During anagen to catagen transition, HFs express higher TGF- $\beta 1$, which suppresses proliferation and increases apoptosis in HFs [125]. HFs might induce adipocyte dedifferentiation through TGF- $\beta 1$ signaling in catagen [126].

Adipose tissue has been shown to exhibit non-metabolic functions [127]. In bone marrow, hematopoiesis and hematopoietic stem cell activity are suppressed when more mature adipocytes are present $[128,129]$. In skin, adipocytes and preadipocytes show opposite roles in the regulation of HFSC activity during the physiological hair cycling (Fig. 2) [127]. Immediately after HFSCs are activated to initiate anagen growth, mature adipocytes release BMP proteins to suppress the activity of HFSCs [28]. This might prevent overactivation of HFSCs by consolidating quiescence. On the other hand, during the transition from telogen to anagen, preadipocytes stimulate HFSCs through paracrine secretion of PDGF [27]. The reciprocal signaling and intimate interaction between HFs and adipose tissue highlight the interdependence between HFSCs and its niche cells to maintain appropriate tissue dynamics in skin.
In addition to passive fat storage, adipose tissue also exhibits other non-metabolic functions [127]. During bacterial invasion, cutaneous adipocytes undergo reactive adipogenesis to increase the production of antimicrobial peptides against bacteria [130]. We speculate that adipocytes in the HFSC niche might play a role in sensing environmental changes, such systemic nutritional states or local skin injury. Hair growth is affected by the systemic nutritional states [131]. In human, impaired hair growth is observed in individuals with protein/energy malnutrition [132]. HFs can be arrested in prolonged telogen during experimental calorie restriction [5, 133]. How HFSCs detect the systemic nutritional states is unclear. One possibility is that HFSCs can directly sense the systemic nutritional changes. mTOR signaling is a key pathway for metabolic response to the nutritional state [134], and upregulated mTOR signaling is essential for HFSC activation in the early anagen and regeneration following ionizing radiation injury $[135,136]$. HFSCs might tune its mTOR signaling according to the changes of systemic nutrition. The other possibility is that the nutritional states are detected by niche cells, such as adipocytes. In the intestine, calorie restriction reduces mTOR activity in the niche Paneth cells [137]. Subsequently, Paneth cells signal to intestinal SCs to increase intestinal SC numbers. Clinical observation suggests that obesity might negatively affect hair growth [138]. Adipocytes might regulate HFSCs through the release of adipokines according the systemic nutritional states [127].

\section{Signals from sensory nerves and message-relaying function of sympathetic nerves to activate HFSCs via an ipRGC-SCN-sympathetic circuit}

HF is a highly innervated sensory organ. The nonencapsulated endings of sensory nerve surround HFs for the mechanosensory function $[139,140]$. In HFs, sensory nerves innervate upper bulge to form the sensory piloneural niche (Fig. 2) [139, 140]. Through secreting SHH ligands, this sensory piloneural niche maintains higher hedgehog signaling activity in the HFSCs of the upper bulge region [26]. Although this sensory piloneural niche does not significantly affect hair regeneration, the ability of the upper bulge cells to repair epidermal injury is dependent on the sustained upregulation of hedgehog signaling [26].

The piloerection function of HFs relies on the ordered integration of sympathetic nerves and arrector pili muscle around HFs. Sympathetic nerves not only densely surround arrector pili muscle but also loop around HFSCs [34, 140]. It is intriguing whether sympathetic nerves around the HFs have dual roles in both piloerection (goosebumps) and HFSC regulation. In the 
bone marrow, sympathetic nerves control multiple functions of hematopoietic SCs, including their mobilization, maintenance of young functional signature and regeneration from chemotherapeutic injury [141-143]. Sympathetic nerves also transduce the central circadian rhythms to hematopoietic SCs for their daily rhythmic oscillating egress from the bone marrow [143-145]. Clinical observation showed that hypertrichosis in the form of "hemitrichosis" can be a result of sympathetic nerve hyperactivity due to thoracic surgical injury [145], suggesting a stimulating effect of sympathetic nerves to hair growth. Early experiments suggested that sympathetic nerves might promote anagen progression after HFSCs are activated in the physiological state [140]. We found that light can stimulate hair growth not only directly through cutaneous irradiation but also indirectly through the eyes $[34,43,146]$. Light irradiation to murine eyes, a danger signal to nocturnal animals, is detected by the non-conventional photoreceptor melanopsin of intrinsically photosensitive retinal ganglion cells (ipRGCs) (Fig. 4) [34]. Light signals are transmitted via ipRGCs to the suprachiasmatic nucleus to activate the systemic sympathetic system. A high sympathetic tone increases local norepinephrine release which subsequently upregulates hedgehog signaling in HFSCs, promoting their activation. Therefore, sympathetic nerves are the niche gateway for internal HFSCs to communicate with the external world by relaying the external light signals to the HFSC niche. Therapeutically, stimulating adrenergic receptors of HFSCs can be a way to promote hair growth.

\section{Conclusion}

Since cyclic hair regeneration can be easily observed, the HF has become a favored model to explore how tissue stem cell activities are regulated. Accumulative results have helped to identify various component cells of the HFSC niche and to elucidate how these niche cells influence HFSCs. The integration of functional distinctive niche modules, such as signaling, sensing and message-relaying modules, has added the complexity of HFSC regulation and also allows HFSCs to interact with the local, systemic and external environments to adapt their activity for tissue needs. Pathological changes of the HFSC niche can lead to dysregulated hair growth or HFSC loss in diseased states. Studying how HFSCs are regulated by the niche in the physiological and diseased states can uncover new therapeutic targets to prevent hair loss as well as to promote hair regeneration.

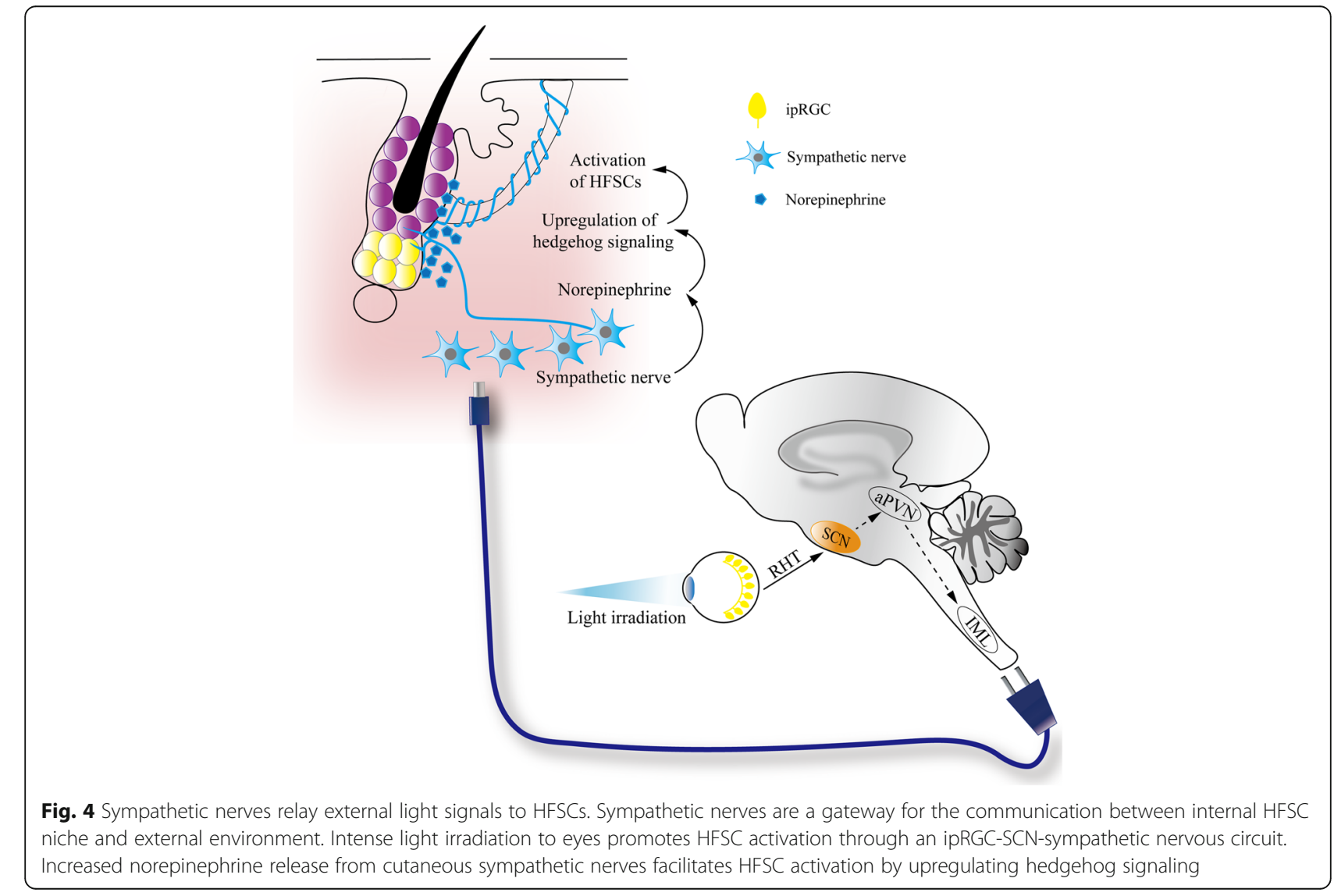




\section{Acknowledgements}

Not applicable.

\section{Authors' contributions}

CLC, WYH, EHCW, KYT and SJL wrote the manuscript. All authors read and approved the final manuscript.

\section{Funding}

This work was supported by Taiwan Bio-Development Foundation (TBF) (to SJ.L), Taiwan Ministry of Science and Technology (MOST107-2314-B-002-064-MY3; MOST108-2811-B-002-583), National Taiwan University Hospital (UN108-029, 107S3781, 108-T17, 109-T17) and Taiwan National Health Research Institutes (NHRI-EX108-10811EI, NHRI-EX109-10811El).

\section{Availability of data and materials}

Not applicable.

\section{Ethics approval and consent to participate}

Not applicable.

\section{Consent for publication}

Not applicable.

\section{Competing interests}

The authors declare that they have no competing interests.

\section{Author details}

'Department of Biomedical Engineering, College of Medicine and College of Engineering, National Taiwan University, Taipei, Taiwan. ${ }^{2}$ Department of Dermatology, Columbia University, New York, NY, USA. ${ }^{3}$ Genome and Systems Biology Degree Program, National Taiwan University and Academia Sinica, Taipei, Taiwan. ${ }^{4}$ Department of Dermatology, National Taiwan University Hospital and National Taiwan University College of Medicine, Taipei, Taiwan. ${ }^{5}$ Research Center for Developmental Biology and Regenerative Medicine, National Taiwan University, Taipei, Taiwan. ${ }^{6}$ Graduate Institute of Clinical Medicine, College of Medicine, National Taiwan University, Taipei, Taiwan.

\section{Received: 15 October 2019 Accepted: 23 January 2020}

Published online: 14 March 2020

\section{References}

1. McGarvey EL, et al. Psychological sequelae and alopecia among women with cancer. Cancer Pract. 2001;9(6):283-9.

2. Cotsarelis G, Sun T, Lavker RM. Label-retaining cells reside in the bulge area of pilosebaceous unit: implications for follicular stem cells, hair cycle, and skin carcinogenesis. Cell. 1990;61(7):1329-37.

3. Greco V, et al. A two-step mechanism for stem cell activation during hair regeneration. Cell Stem Cell. 2009;4(2):155-69.

4. Hsu YC, Pasolli HA, Fuchs E. Dynamics between stem cells, niche, and progeny in the hair follicle. Cell. 2011;144(1):92-105.

5. Chase HB. Growth of the hair. Physiol Rev. 1954;34(1):113-26.

6. Chen CC, et al. The Modulatable stem cell niche: tissue interactions during hair and feather follicle regeneration. J Mol Biol. 2016;428(7):1423-40.

7. Hsu YC, Li L, Fuchs E. Emerging interactions between skin stem cells and their niches. Nat Med. 2014;20(8):847-56.

8. Muller-Rover $\mathrm{S}$, et al. A comprehensive guide for the accurate classification of murine hair follicles in distinct hair cycle stages. J Invest Dermatol. 2001; 117(1):3-15.

9. Dry FW. The coat of the mouse (Mus musculus). J Genet. 1926;16(3):54

10. Paus R, Cotsarelis G. The biology of hair follicles. N Engl J Med. 1999;341(7): 491-7.

11. Rahmani W, et al. Hair follicle dermal stem cells regenerate the dermal sheath, repopulate the dermal papilla, and modulate hair type. Dev Cell. 2014;31(5):543-58.

12. Mesa KR, et al. Niche-induced cell death and epithelial phagocytosis regulate hair follicle stem cell pool. Nature. 2015;522(7554):94-7.

13. Rompolas $P$, et al. Live imaging of stem cell and progeny behaviour in physiological hair-follicle regeneration. Nature. 2012;487(7408):496-9.
14. Enshell-Seijffers D, et al. Beta-catenin activity in the dermal papilla of the hair follicle regulates pigment-type switching. Proc Natl Acad Sci U S A. 2010;107(50):21564-9.

15. Jahoda CA, Horne KA, Oliver RF. Induction of hair growth by implantation of cultured dermal papilla cells. Nature. 1984;311(5986):560-2.

16. Oshimori N, Fuchs E. Paracrine TGF-beta signaling counterbalances BMPmediated repression in hair follicle stem cell activation. Cell Stem Cell. 2012; 10(1):63-75

17. Myung PS, et al. Epithelial Wnt ligand secretion is required for adult hair follicle growth and regeneration. J Invest Dermatol. 2013;133(1):31-41.

18. Rezza A, et al. Signaling networks among stem cell precursors, transitamplifying progenitors, and their niche in developing hair follicles. Cell Rep. 2016:14(12):3001-18

19. Rendl M, Polak L, Fuchs E. BMP signaling in dermal papilla cells is required for their hair follicle-inductive properties. Genes Dev. 2008; 22(4):543-57.

20. Blanpain C, et al. Self-renewal, multipotency, and the existence of two cell populations within an epithelial stem cell niche. Cell. 2004;1 18(5):635-48.

21. Oshima $\mathrm{H}$, et al. Morphogenesis and renewal of hair follicles from adult multipotent stem cells. Cell. 2001;104(2):233-45.

22. Tumbar T, et al. Defining the epithelial stem cell niche in skin. Science. 2004; 303(5656):359-63.

23. Hsu YC, Li L, Fuchs E. Transit-amplifying cells orchestrate stem cell activity and tissue regeneration. Cell. 2014;157(4):935-49.

24. Chuong CM, Cotsarelis G, Stenn K. Defining hair follicles in the age of stem cell bioengineering. J Invest Dermatol. 2007;127(9):2098-100.

25. Pena-Jimenez $D$, et al. Lymphatic vessels interact dynamically with the hair follicle stem cell niche during skin regeneration in vivo. EMBO J. 2019;38(19):e101688.

26. Brownell I, et al. Nerve-derived sonic hedgehog defines a niche for hair follicle stem cells capable of becoming epidermal stem cells. Cell Stem Cell. 2011;8(5):552-65.

27. Festa $\mathrm{E}$, et al. Adipocyte lineage cells contribute to the skin stem cell niche to drive hair cycling. CELL. 2011;146(5):761-71.

28. Plikus MV, et al. Cyclic dermal BMP signalling regulates stem cell activation during hair regeneration. Nature. 2008;451(7176):340-4.

29. Wang ECE, et al. A subset of TREM2(+) dermal macrophages secretes oncostatin M to maintain hair follicle stem cell quiescence and inhibit hair growth. Cell Stem Cell. 2019;24(4):654-69 e6.

30. Castellana D, Paus R, Perez-Moreno M. Macrophages contribute to the cyclic activation of adult hair follicle stem cells. PLoS Biol. 2014;12(12):e1002002.

31. Ali N, et al. Regulatory T cells in skin facilitate epithelial stem cell differentiation. Cell. 2017;169(6):1119-29 e11.

32. Paus R, et al. Mast cell involvement in murine hair growth. Dev Biol. 1994; 163(1):230-40.

33. Botchkarev VA, et al. Hair cycle-dependent changes in adrenergic skin innervation, and hair growth modulation by adrenergic drugs. J Invest Dermatol. 1999;113(6):878-87.

34. Fan SM-Y, et al. External light activates hair follicle stem cells through eyes via an ipRGC-SCN-sympathetic neural pathway. Proc Natl Acad Sci U S A. 2018;115(29):E6880-9.

35. Chen CC, et al. Organ-level quorum sensing directs regeneration in hair stem cell populations. Cell. 2015;161(2):277-90.

36. Yu Z, et al. Hoxc-dependent mesenchymal niche heterogeneity drives regional hair follicle regeneration. Cell Stem Cell. 2018;23(4):487-500 e6.

37. Plikus MV. New activators and inhibitors in the hair cycle clock: targeting stem cells' state of competence. J Invest Dermatol. 2012;132(5):1321-4.

38. Murray PJ, et al. Modelling hair follicle growth dynamics as an excitable medium. PLoS Comput Biol. 2012;8(12):e1002804.

39. Plikus MV, et al. Self-organizing and stochastic behaviors during the regeneration of hair stem cells. Science. 2011;332(6029):586-9.

40. Choi YS, et al. Distinct functions for Wnt/beta-catenin in hair follicle stem cell proliferation and survival and interfollicular epidermal homeostasis. Cell Stem Cell. 2013;13(6):720-33.

41. Leishman $\mathrm{E}$, et al. Foxp1 maintains hair follicle stem cell quiescence through regulation of Fgf18. Development. 2013;140(18):3809-18.

42. Wang Q, et al. A multi-scale model for hair follicles reveals heterogeneous domains driving rapid spatiotemporal hair growth patterning. Elife. 2017;6: e22772.

43. Sheen YS, et al. Visible red light enhances physiological anagen entry in vivo and has direct and indirect stimulative effects in vitro. Lasers Surg Med. 2015:47(1):50-9. 
44. Wu YF, et al. Enhancing hair follicle regeneration by nonablative fractional laser: assessment of irradiation parameters and tissue response. Lasers Surg Med. 2015:47(4):331-41.

45. Osaka N, et al. ASK1-dependent recruitment and activation of macrophages induce hair growth in skin wounds. J Cell Biol. 2007;176(7):903-9.

46. Sennett R, Rendl M. Mesenchymal-epithelial interactions during hair follicle morphogenesis and cycling. Semin Cell Dev Biol. 2012;23(8):917-27.

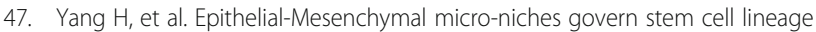
choices. Cell. 2017;169(3):483-96 e13.

48. Enshell-Seijffers D, et al. Beta-catenin activity in the dermal papilla regulates morphogenesis and regeneration of hair. Dev Cell. 2010;18(4):633-42.

49. Huang WY, et al. Mobilizing transit-amplifying cell-derived ectopic progenitors prevents hair loss from chemotherapy or radiation therapy. Cancer Res. 2017;77(22):6083-96.

50. Huang $W Y$, et al. Anagen hair follicle repair: timely regenerative attempts from plastic extra-bulge epithelial cells. Exp Dermatol. 2019;28(4):406-12.

51. Marshall WA, Tanner JM. Variations in pattern of pubertal changes in girls. Arch Dis Child. 1969;44(235):291-303.

52. Marshall WA, Tanner JM. Variations in the pattern of pubertal changes in boys. Arch Dis Child. 1970;45(239):13-23.

53. Nyholt DR, et al. Genetic basis of male pattern baldness. J Invest Dermatol. 2003;121(6):1561-4.

54. Azziz R. The evaluation and management of hirsutism. Obstet Gynecol. 2003;101(5 Pt 1):995-1007.

55. Thornton MJ, et al. Differences in testosterone metabolism by beard and scalp hair follicle dermal papilla cells. Clin Endocrinol. 1993;39(6):633-9.

56. Randall VA, et al. The hair follicle: a paradoxical androgen target organ. Horm Res. 2000;54(5-6):243-50.

57. Hibberts NA, Howell AE, Randall VA. Balding hair follicle dermal papilla cells contain higher levels of androgen receptors than those from non-balding scalp. J Endocrinol. 1998;156(1):59-65.

58. Ando $Y$, et al. Expression of mRNA for androgen receptor, 5alpha-reductase and 17beta-hydroxysteroid dehydrogenase in human dermal papilla cells. $\mathrm{Br}$ J Dermatol. 1999;141(5):840-5.

59. Choudhry $R$, et al. Localization of androgen receptors in human skin by immunohistochemistry: implications for the hormonal regulation of hair growth, sebaceous glands and sweat glands. J Endocrinol. 1992;133(3): 467-75.

60. Lai JJ, et al. The role of androgen and androgen receptor in skin-related disorders. Arch Dermatol Res. 2012;304(7):499-510.

61. Courtois M, et al. Ageing and hair cycles. Br J Dermatol. 1995;132(1):86-93.

62. Matsumura $\mathrm{H}$, et al. Hair follicle aging is driven by transepidermal elimination of stem cells via COL17A1 proteolysis. Science. 2016;351(6273): aad4395.

63. Chen W, Zouboulis CC, Orfanos CE. The 5 alpha-reductase system and its inhibitors. Recent development and its perspective in treating androgendependent skin disorders. Dermatology. 1996;193(3):177-84.

64. Bahta AW, et al. Premature senescence of balding dermal papilla cells in vitro is associated with p16(INK4a) expression. J Invest Dermatol. 2008; 128(5):1088-94.

65. Randall VA, Hibberts NA, Hamada K. A comparison of the culture and growth of dermal papilla cells from hair follicles from non-balding and balding (androgenetic alopecia) scalp. Br J Dermatol. 1996;134(3):437-44.

66. Huang WY, et al. Stress-induced premature senescence of dermal papilla cells compromises hair follicle epithelial-mesenchymal interaction. J Dermatol Sci. 2017;86(2):114-22.

67. Kwack MH, et al. Dihydrotestosterone-inducible IL-6 inhibits elongation of human hair shafts by suppressing matrix cell proliferation and promotes regression of hair follicles in mice. J Invest Dermatol. 2012;132(1):43-9.

68. Kwack $\mathrm{MH}$, et al. Dihydrotestosterone-inducible dickkopf 1 from balding dermal papilla cells causes apoptosis in follicular keratinocytes. J Invest Dermatol. 2008;128(2):262-9.

69. Inui $\mathrm{S}$, et al. Identification of androgen-inducible TGF-beta1 derived from dermal papilla cells as a key mediator in androgenetic alopecia. J Investig Dermatol Symp Proc. 2003;8(1):69-71.

70. Paus $R$, et al. Transforming growth factor-beta receptor type I and type | expression during murine hair follicle development and cycling. J Invest Dermatol. 1997;109(4):518-26.

71. Turksen K, et al. Interleukin 6: insights to its function in skin by overexpression in transgenic mice. Proc Natl Acad Sci U S A. 1992:89(11): 5068-72.
72. McConnell JD, et al. Finasteride, an inhibitor of 5 alpha-reductase, suppresses prostatic dihydrotestosterone in men with benign prostatic hyperplasia. J Clin Endocrinol Metab. 1992;74(3):505-8.

73. Bramson HN, et al. Unique preclinical characteristics of GG745, a potent dual inhibitor of 5AR. J Pharmacol Exp Ther. 1997;282(3):1496-502.

74. Kaufman KD, et al. Finasteride in the treatment of men with androgenetic alopecia. Finasteride male pattern hair loss study group. J Am Acad Dermatol. 1998;39(4 Pt 1):578-89.

75. Olsen EA, et al. The importance of dual 5alpha-reductase inhibition in the treatment of male pattern hair loss: results of a randomized placebocontrolled study of dutasteride versus finasteride. J Am Acad Dermatol. 2006;55(6):1014-23.

76. Rossi A, et al. Minoxidil use in dermatology, side effects and recent patents. Recent Patents Inflamm Allergy Drug Discov. 2012;6(2):130-6.

77. De Villez RL. Topical minoxidil therapy in hereditary androgenetic alopecia. Arch Dermatol. 1985;121(2):197-202.

78. Zappacosta AR. Reversal of baldness in patient receiving minoxidil for hypertension. N Engl J Med. 1980;303(25):1480-1.

79. Headington JT. Hair follicle biology and topical minoxidil: possible mechanisms of action. Dermatologica. 1987;175(Suppl 2):19-22.

80. Kumamoto $T$, et al. Hair follicles serve as local reservoirs of skin mast cell precursors. Blood. 2003;102(5):1654-60.

81. Paus $R$, et al. The hair follicle and immune privilege. J Investig Dermatol Symp Proc. 2003;8(2):188-94

82. Maurer M, Paus R, Czarnetzki BM. Mast cells as modulators of hair follicle cycling. Exp Dermatol. 1995;4(4 Pt 2):266-71.

83. Maurer $\mathrm{M}$, et al. The mast cell products histamine and serotonin stimulate and TNF-alpha inhibits the proliferation of murine epidermal keratinocytes in situ. J Dermatol Sci. 1997;16(1):79-84.

84. Hobo A, et al. IL-17-positive mast cell infiltration in the lesional skin of lichen planopilaris: Possible role of mast cells in inducing inflammation and dermal fibrosis in cicatricial alopecia. Exp Dermatol. 2020;29(3):273-77.

85. Xu X, et al. Scarring alopecia associated with mastocytosis. J Cutan Pathol. 2003;30(9):561-5.

86. Michel $L$, et al. Study of gene expression alteration in male androgenetic alopecia: evidence of predominant molecular signalling pathways. $\mathrm{Br} \mathrm{J}$ Dermatol. 2017;177(5):1322-36.

87. Jameson J, Havran WL. Skin gammadelta T-cell functions in homeostasis and wound healing. Immunol Rev. 2007;215(1):114-22.

88. Lee $\mathrm{P}$, et al. Stimulation of hair follicle stem cell proliferation through an IL-1 dependent activation of $\gamma \delta T$-cells. eLife. 2017;6:366.

89. Paus $\mathrm{R}$, et al. Generation and cyclic remodeling of the hair follicle immune system in mice. J Invest Dermatol. 1998;111(1):7-18.

90. Harel S, et al. Pharmacologic inhibition of JAK-STAT signaling promotes hair growth. Sci Adv. 2015;1(9):e1500973.

91. Wang $X$, Chen $H$, Tian $R$, et al. Macrophages induce AKT/ $\beta$-catenindependent Lgr5+ stem cell activation and hair follicle regeneration through TNF. Nat Commun. 2017;8:14091

92. Chu S-Y, et al. Mechanical stretch induces hair regeneration through the alternative activation of macrophages. Nat Commun. 2019;10(1):1524.

93. Gilhar A, Paus R, Kalish RS. Lymphocytes, neuropeptides, and genes involved in alopecia areata. J Clin Invest. 2007;117(8):2019-27.

94. Harries MJ, et al. Lichen planopilaris is characterized by immune privilege collapse of the hair follicle's epithelial stem cell niche. J Pathol. 2013;231(2):236-47.

95. Anzai $A$, et al. Pathomechanisms of immune-mediated alopecia. Int Immunol. 2019;31(7):439-47.

96. Gregoriou S, et al. Cytokines and other mediators in alopecia areata. Mediat Inflamm. 2010;2010:928030.

97. Strazzulla LC, et al. Alopecia areata: disease characteristics, clinical evaluation, and new perspectives on pathogenesis. J Am Acad Dermatol. 2018;78(1):1-12

98. Wang E, McElwee KJ. Etiopathogenesis of alopecia areata: why do our patients get it? Dermatol Ther. 2011;24(3):337-47.

99. Wang EHC, et al. Identification of autoantigen epitopes in alopecia areata. J Invest Dermatol. 2016;136(8):1617-26.

100. Gilhar A, et al. Melanocyte-associated T cell epitopes can function as autoantigens for transfer of alopecia areata to human scalp explants on Prkdc(scid) mice. J Invest Dermatol. 2001:117(6):1357-62.

101. de Jong A, et al. High-throughput T cell receptor sequencing identifies clonally expanded CD8+ T cell populations in alopecia areata. JCI Insight 2018;3(19):e121949. 
102. Strazzulla LC, et al. Alopecia areata: an appraisal of new treatment approaches and overview of current therapies. J Am Acad Dermatol. 2018; 78(1):15-24

103. Gilhar A, Etzioni A, Paus R. Alopecia areata. N Engl J Med. 2012;366(16): $1515-25$.

104. Cranwell WC, et al. Treatment of alopecia areata: an Australian expert consensus statement. Australas J Dermatol. 2019;60(2):163-70.

105. Alkhalifah A, et al. Alopecia areata update: part II. Treatment. J Am Acad Dermatol. 2010;62(2):191-202 quiz 203-4.

106. Cotellessa C, et al. The use of topical diphenylcyclopropenone for the treatment of extensive alopecia areata. J Am Acad Dermatol. 2001;44(1):73-6.

107. Petukhova L, et al. Genome-wide association study in alopecia areata implicates both innate and adaptive immunity. Nature. 2010;466(7302): $113-7$.

108. Paus R, Bulfone-Paus S, Bertolini M. Hair follicle immune privilege revisited: the key to alopecia Areata management. J Investig Dermatol Symp Proc. 2018;19(1):S12-7.

109. Castela $E$, et al. Effects of low-dose recombinant interleukin 2 to promote Tregulatory cells in alopecia areata. JAMA Dermatol. 2014;150(7):748-51.

110. Xing $L$, et al. Alopecia areata is driven by cytotoxic $T$ lymphocytes and is reversed by JAK inhibition. Nat Med. 2014;20(9):1043-9.

111. Betz RC, et al. Genome-wide meta-analysis in alopecia areata resolves HLA associations and reveals two new susceptibility loci. Nat Commun. 2015;6:5966.

112. Mackay-Wiggan J, et al. Oral ruxolitinib induces hair regrowth in patients with moderate-to-severe alopecia areata. JCI Insight. 2016;1(15):e89790.

113. Jabbari $\mathrm{A}$, et al. Treatment of an alopecia areata patient with tofacitinib results in regrowth of hair and changes in serum and skin biomarkers. Exp Dermatol. 2016;25(8):642-3.

114. Wang EHC, et al. JAK inhibitors for treatment of alopecia Areata. J Invest Dermatol. 2018;138(9):1911-6.

115. Mehregan DA, Van Hale HM, Muller SA. Lichen planopilaris: clinical and pathologic study of forty-five patients. J Am Acad Dermatol. 1992;27(6 Pt 1): 935-42.

116. Kang H, et al. Lichen planopilaris. Dermatol Ther. 2008;21(4):249-56.

117. Imanishi $\mathrm{H}$, et al. Epithelial-to-Mesenchymal stem cell transition in a human organ: lessons from lichen Planopilaris. J Invest Dermatol. 2018;138(3):511-9.

118. Spencer LA, Hawryluk EB, English JC 3rd. Lichen planopilaris: retrospective study and stepwise therapeutic approach. Arch Dermatol. 2009;145(3):333-4.

119. Chase HB, Montagna W, Malone JD. Changes in the skin in relation to the hair growth cycle. Anat Rec. 1953;116(1):75-81.

120. Hansen LS, et al. The influence of the hair cycle on the thickness of mouse skin. Anat Rec. 1984;210(4):569-73.

121. Nicu C, et al. Do human dermal adipocytes switch from lipogenesis in anagen to lipophagy and lipolysis during catagen in the human hair cycle? Exp Dermatol. 2019;28(4):432-5.

122. Rodeheffer MS, Birsoy K, Friedman JM. Identification of white adipocyte progenitor cells in vivo. CELL. 2008;135(2):240-9.

123. Donati $\mathrm{G}$, et al. Epidermal Wnt/beta-catenin signaling regulates adipocyte differentiation via secretion of adipogenic factors. Proc Natl Acad Sci U S A. 2014;111(15):E1501-9.

124. Zhang B, et al. Hair follicles' transit-amplifying cells govern concurrent dermal adipocyte production through sonic hedgehog. Genes Dev. 2016; 30(20):2325-38.

125. Foitzik K, et al. Control of murine hair follicle regression (catagen) by TGFbeta1 in vivo. FASEB J. 2000;14(5):752-60.

126. Cote JA, et al. Role of the TGF-beta pathway in dedifferentiation of human mature adipocytes. Febs Open Bio. 2017;7(8):1092-101.

127. Guerrero-Juarez CF, Plikus MV. Emerging nonmetabolic functions of skin fat. Nat Rev Endocrinol. 2018;14(3):163-73.

128. Ambrosi TH, et al. Adipocyte Accumulation in the Bone Marrow during Obesity and Aging Impairs Stem Cell-Based Hematopoietic and Bone Regeneration. Cell Stem Cell. 2017;20(6):771-784.e6.

129. Naveiras $\mathrm{O}$, et al. Bone-marrow adipocytes as negative regulators of the haematopoietic microenvironment. Nature. 2009;460(7252):259-63.

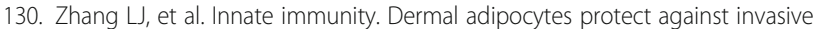
Staphylococcus aureus skin infection. Science. 2015;347(6217):67-71.

131. Goldberg L, Lenzy Y. Nutrition and hair. Clin Dermatol. 2010;28(4):412-9.

132. Lucas $D$, et al. Norepinephrine reuptake inhibition promotes mobilization in mice: potential impact to rescue low stem cell yields. Blood. 2012;119(17):3962-5.
133. Chow A, et al. Bone marrow CD169+ macrophages promote the retention of hematopoietic stem and progenitor cells in the mesenchymal stem cell niche. J Exp Med. 2011;208(2):261-71.

134. Saxton RA, Sabatini DM. mTOR signaling in growth, metabolism, and disease. Cell. 2017;169(2):361-71.

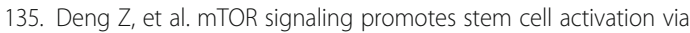
counterbalancing BMP-mediated suppression during hair regeneration. Mol Cell Biol. 2015;7(1):62-72.

136. Wang WH, et al. Activation of $\mathrm{mTORC1}$ signaling is required for timely hair follicle regeneration from radiation injury. Radiat Res. 2017;188(6):681-9.

137. Yilmaz $\mathrm{OH}$, et al. mTORC1 in the Paneth cell niche couples intestinal stemcell function to calorie intake. Nature. 2012;486(7404):490-5.

138. Yang CC, et al. Higher body mass index is associated with greater severity of alopecia in men with male-pattern androgenetic alopecia in Taiwan: a cross-sectional study. J Am Acad Dermatol. 2014;70(2):297-302 e1.

139. Yilmaz $\mathrm{OH}$, et al. mTORC1 in the Paneth cell niche couples intestinal stemcell function to calorie intake. Nature. 2012;486(7404):490-95.

140. Botchkarev VA, et al. Hair cycle-dependent plasticity of skin and hair follicle innervation in normal murine skin. J Comp Neurol. 1997;386(3):379-95.

141. Maryanovich $M$, et al. Adrenergic nerve degeneration in bone marrow drives aging of the hematopoietic stem cell niche. Nat Med. 2018;24(6):782-91.

142. Lucas $D$, et al. Chemotherapy-induced bone marrow nerve injury impairs hematopoietic regeneration. Nat Med. 2013;19(6):695-703.

143. Katayama $Y$, et al. Signals from the sympathetic nervous system regulate hematopoietic stem cell egress from bone marrow. Cell. 2006;124(2):407-21.

144. Lucas D, et al. Mobilized hematopoietic stem cell yield depends on speciesspecific circadian timing. Cell Stem Cell. 2008;3(4):364-6.

145. Mendez-Ferrer $S$, et al. Haematopoietic stem cell release is regulated by circadian oscillations. Nature. 2008;452(7186):442-7.

146. Mai-Yi Fan S, et al. Efficacy and safety of a low-level light therapy for androgenetic alopecia: a 24-week, randomized, double-blind, self-comparison, sham device-controlled trial. Dermatol Surg. 2018;44(11):1411-20.

\section{Publisher's Note}

Springer Nature remains neutral with regard to jurisdictional claims in published maps and institutional affiliations.

Ready to submit your research? Choose BMC and benefit from:

- fast, convenient online submission

- thorough peer review by experienced researchers in your field

- rapid publication on acceptance

- support for research data, including large and complex data types

- gold Open Access which fosters wider collaboration and increased citations

- maximum visibility for your research: over $100 \mathrm{M}$ website views per year

At BMC, research is always in progress.

Learn more biomedcentral.com/submissions 